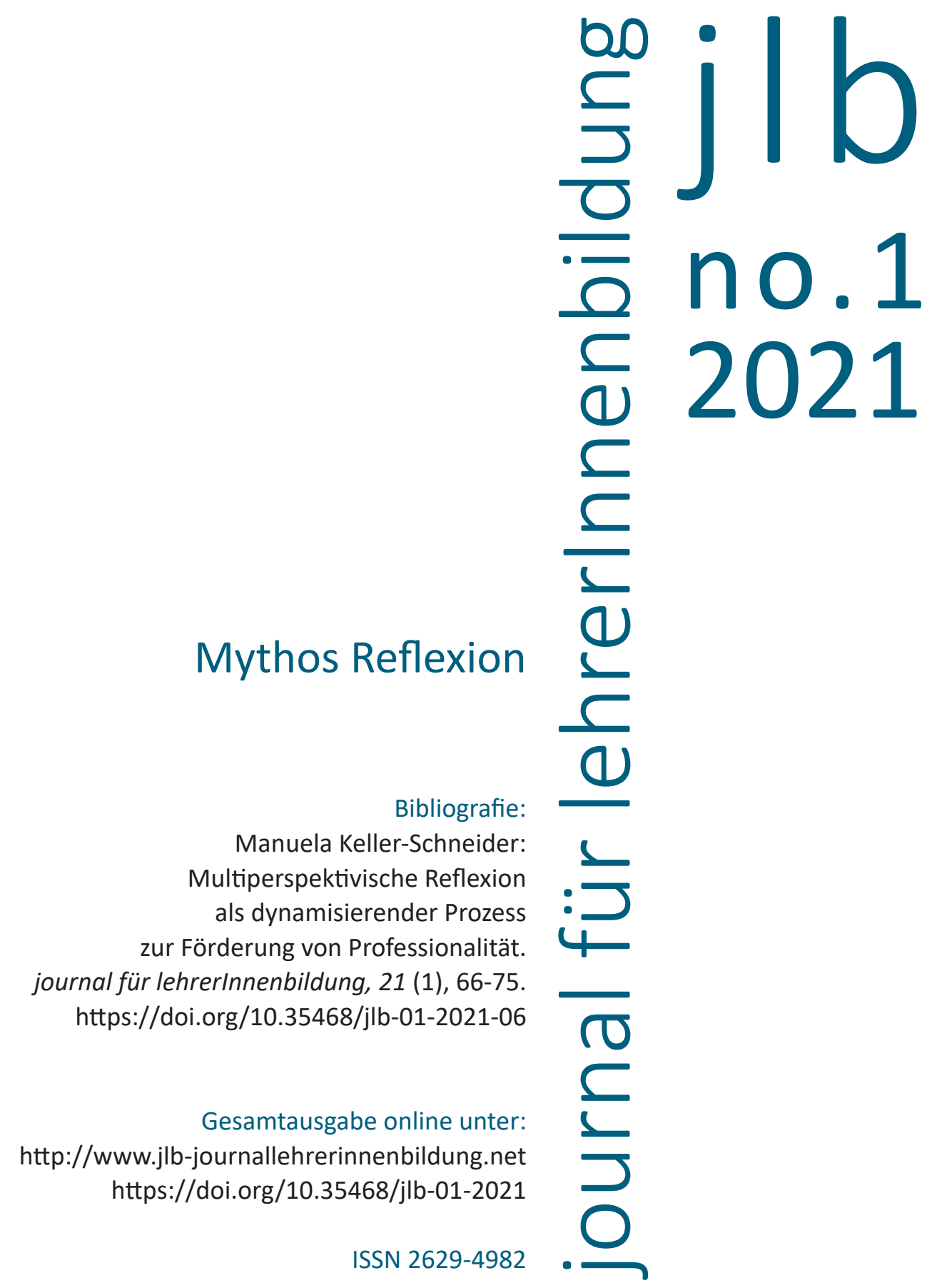


06 Manuela Keller-Schneider

\section{Multiperspektivische Reflexion als dynamisierender Prozess zur Förderung von Professionalität}


Professionalität von Lehrpersonen zeichnet sich durch erworbene Expertise aus, wobei insbesondre eine adaptive Expertise eine auf situationsspezifische Anforderungen ausgerichtete Bewältigung von Anforderungen ermöglicht. Der Beitrag zeigt theoriegestützt auf, dass eine adaptive Expertise über die Auseinandersetzung mit herausfordernden Situationen aufgebaut wird und dass unterschiedliche Komponenten der individuellen Ressourcen dabei von Bedeutung sind. Darauf aufbauend wird die Arbeit mit einem auf diese Komponenten ausgerichteten Reflexionsinstrument vorgestellt, mit welchem eine subjektiv wahrgenommene Situation analysiert wird, um an die unterschiedlichen Komponenten anknüpfend neue Sichtweisen und Lösungsmöglichkeiten abzuleiten.

\section{Aufbau von Erkenntnissen über die Reflexion von Erfahrung}

Reflexion fokussiert auf Gedanken und Handlungen, Prozesse und Veränderungen, konzeptuelle Rahmungen sowie auf sich selbst (Nguyen, Fernandez, Karsenti \& Charlin, 2014) und geht damit über Selbstwahrnehmung sowie Selbstaufmerksamkeit hinaus. Im Beruf von Lehrpersonen ist Reflexion unabdingbar (Helsper, 2001), um der Kontingenz pädagogischen Handelns zu begegnen (Combe, Paseka \& Keller-Schneider, 2018). Die Fähigkeit, Ungewissheit als Herausforderung anzunehmen, soll bereits im Studium erworben werden (KellerSchneider, 2018a; Korthagen, 2014), um sich auf die Ungewissheit beruflichen Handelns vorzubereiten. Da Wissen nicht direkt in Handlung überführt werden kann (Mandl \& Gerstenmaier, 2000), sondern als reflection in action (Schön, 1983) ein Abwägen von Handlungsoptionen und situativ bedingter Entscheidungen erfordert (Blömeke, Gustafsson, Shavelson, 2015), stellt die Förderung von Reflexionskompetenz als reflection on action (Schön, 1983) eine zentrale Anforderung in der Lehrpersonenbildung dar.

In spezifischen Situationen erforderliche Handlungsentscheidungen werden von kognitiven und emotional-motivationalen Ressourcen sowie von Wahrnehmungs- und Deutungsprozessen mitbestimmt (Blömeke et al., 2015; Keller-Schneider, 2020a). Sie prägen die Art und Weise der Bewältigung von Anforderungen und damit auch die daraus 
hervorgehenden Erfahrungen und Erkenntnisse mit, die als subjektives Wissen (Neuweg, 2014) in die individuellen Ressourcen integriert werden und diese nicht nur erweitern, sondern auch transformieren (Dreyfus \& Dreyfus, 1986; Berliner, 2001).

Professionalisierung als kontinuierlicher Prozess erfolgt in der Auseinandersetzung mit sich stellenden Anforderungen, der Reflexion von Erfahrungen und der Integration von daraus hervorgehenden Erkenntnissen. Erfahrung und deren Reflexion werden damit zur Ressource für die Gewinnung von Erkenntnissen. Über die Bildung von Synergien (chunks, Gruber \& Degner, 2016) ermöglicht Professionalisierung eine Reduktion der Komplexität situativer Anforderungen, sowie adaptive Expertise (Tartwijk, Zwart \& Wubbels, 2017) und den Aufbau einer beruflichen Identität (Cherrington, 2017). Adaptive Expertise zeigt sich im Handeln und ermöglicht, relevante Faktoren zu erkennen und das eigene Handeln auf die Situation auszurichten, worin sie sich von routinierter Expertise unterscheidet (Tartwijk et al., 2017).

Die Reflexion von situativ sich stellenden Herausforderungen ermöglicht ein auf die Situation ausgerichtetes, d. h. adaptives Handeln. Doch situativ sich stellende Anforderungen werden individuell verschieden wahrgenommen, bedingt durch die individuell geprägten Ressourcen, die aus Erfahrungen und Erkenntnisse hervorgehen. Die subjektiv geprägte Wahrnehmung und Deutung von Situationen und die daraus hervorgehenden individuell gewichteten Anforderungen sind für Professionalisierungsprozesse zentral, wobei damit deutlich wird, dass auch Professionalisierungsprozesse interindividuell different verlaufen. Dies wird im folgenden Abschnitt stress- und ressourcentheoretisch begründet.

\section{Wahrnehmung und Deutung von Anforderungen mittels individueller Ressourcen}

Stresstheoretischen Ansätzen folgend (Lazarus \& Folkman, 1984) werden Anforderungen unbewusst nach ihrer Bedeutsamkeit und Bewältigbarkeit eingeschätzt, wobei mehrere Komponenten der individuellen Ressourcen und ihr Zusammenwirken dabei von Bedeutung sind (Keller-Schneider, 2020a). Als Herausforderungen angenommene Anforderungen werden bearbeitet und führen zu neuen Erfahrungen 
und Erkenntnissen, welche, in die individuellen Ressourcen integriert, diese transformieren (Gruber \& Degner, 2016). Als Überforderung wahrgenommene Anforderungen können sich in der Vermeidung von Herausforderungen zeigen und zu einer Resistenz Veränderungen gegenüber führen (Tartwijk et al., 2017), wodurch Professionalisierungsprozesse eingeschränkt werden. Subjektiv geprägte Überzeugungen lenken die Wahrnehmung und Bearbeitung von Anforderungen und können vor Überforderungen schützen, schränken damit über Vermeidung der Auseinandersetzung mit Anforderungen die Entwicklung einer adaptiven Expertise ein (vgl. Beispiel unten). Die Bereitschaft, Anforderungen als Herausforderungen anzunehmen und sich mit diesen auseinanderzusetzen, ermöglicht weiterführende Erkenntnisse. In der Reflexion von subjektiv bedeutsamen Erfahrungen können Erkenntnisse gewonnen und unbewusst ablaufende, von Überzeugungen geprägte Prozesse sichtbar und für die weitere Professionalisierung nutzbar gemacht werden.

Der stresstheoretisch hergeleitete Wahrnehmungsprozess wird von mehreren Komponenten der individuellen Ressourcen geprägt. Gestützt auf kompetenz- und ressourcentheoretische Ansätze (Blömeke et al., 2015; Keller-Schneider, 2020a) kommen in der Wahrnehmung und Deutung von Situationen nicht nur Überzeugungen, sondern auch weitere individuelle Ressourcen zum Tragen. Wissen und Können sowie ein positives Konzept eigener Fähigkeiten stellen Voraussetzungen für die Bearbeitung und Bewältigung von Anforderungen dar. Überzeugungen rahmen die Wahrnehmung und bestimmen damit mit, was in den Blick genommen wird und was als nicht relevant oder nicht bewältigbar ausgeklammert wird. Motive und Ziele wirken als dynamisierende Komponenten auf die Bereitschaft und Intensität der Auseinandersetzung. Selbstregulationsfähigkeiten regulieren einzusetzende und zu schützende Ressourcen. Emotionen färben die wahrgenommene Situation affektiv.

Diese im theoriegestützt hergeleiteten Modell der Entwicklung pädagogischer Professionalität hergeleiteten individuellen Ressourcen (Keller-Schneider, 2020a) tragen in ihrem Zusammenwirken zur Wahrnehmung und Deutung sowie zum Umgang mit situativ sich stellenden Anforderungen bei und prägen damit das berufliche Handeln sowie über daraus hervorgehende Erkenntnisse die weitere Professionalisierung. Eine Reflexion von Situationen und Erfahrungen aus den Perspektiven dieser Zugänge kann zu einem differenzierten und 
facettenreichen Verständnis einer Situation führen, eigene Handlungen nach ihrer Angemessenheit durchleuchten sowie weitere Handlungsoptionen eröffnen. Diese Komponenten stellen die Eckpfeiler im nun vorgestellten Reflexionsinstrument dar.

\section{Ein mehrperspektivisches Reflexionsinstrument}

Das professionalisierungstheoretisch begründete Reflexionsinstrument stellt individuell erlebte, $d$. h. reale und für das Subjekt bedeutsame Situationen ins Zentrum und beleuchtet diese aus der Perspektive der unterschiedlichen Komponenten der individuellen Ressourcen, welche die Wahrnehmung, Deutung und Bearbeitung von Situationen mitbestimmen (Keller-Schneider, 2020b). Leitfragen (Abb. 1) erläutern den Fokus der jeweiligen Betrachtungsweise und dienen als Impulse zur Exploration der einzelnen Zugänge.

\begin{tabular}{|c|c|c|}
\hline $\begin{array}{l}\text { Überzeugungen } \\
\text { Wie Lernen Schüler/innen? } \\
\text { Was brauchen sie dazu? } \\
\text { Was kann eine Lehrperson beitragen? } \\
\text { Was ist guter Unterricht? } \\
\text { Was funktioniert? } \\
\ldots\end{array}$ & $\begin{array}{l}\text { Ziele und Motive } \\
\text { Was ist Ihnen wichtig? } \\
\text { Was möchten Sie erreichen? } \\
\text { Was möchten Sie sicher stellen? } \\
\text { Was möchten Sie ermöglichen? } \\
\ldots\end{array}$ & $\begin{array}{l}\text { Selbstregulation, Bereitschaft } \\
\text { Was möchten Sie tun, } \\
\text { um dies zu erreichen? } \\
\text { Wieviel Engagement erwarten Sie } \\
\text { von sich? } \\
\text { Woran erkennen sie, dass es sich lohnt? } \\
\text { Was muss sich zeigen, } \\
\text { damit Sie dran bleiben? }\end{array}$ \\
\hline $\begin{array}{l}\text { Emotionen } \\
\text { Was empfinden Sie? }\end{array}$ & Beschreibung einer konkreten Begebenheit & - Was freut, ärgert, belastet? \\
\hline $\begin{array}{l}\text { Pädagogisch- } \\
\text { psychologisches Wissen } \\
\text { Was wissen Sie aus einer } \\
\text { fachunabhängigen und } \\
\text { fachübergreifenden } \\
\text { Perspektive betrachtet? } \\
\text { Über welches pädagogische, } \\
\text { psychologische und allgemein } \\
\text { didaktische Wissen verfügen Sie? ... }\end{array}$ & $\begin{array}{l}\uparrow \\
\text { Fachwissen } \\
\text { Was wissen Sie über die Sache? } \\
\ldots\end{array}$ & $\begin{array}{l}\text { Fachdidaktisches Wissen } \\
\text { Was wissen Sie aus fachdidaktischer } \\
\text { Perspektive? } \\
\text { Welche Hürden und Schwierigkeiten } \\
\text { gehen von der Sache aus? } \\
\text { Über welches Vorwissen könnten die } \\
\text { Schüler/-innen verfügen? } \\
\text { Wie kann dieses aktiviert und } \\
\text { modifiziert werden? ... }\end{array}$ \\
\hline
\end{tabular}

Abb. 1 Theoriegestützt entwickeltes Reflexionsinstrument zur Analyse alltäglicher Schulsituationen (Keller-Schneider, 2020a, S. 420).

Das Reflexionsinstrument kann von (angehenden) Lehrpersonen in einer Regie oder in supervisorisch angelegten Gruppensitzungen genutzt werden. Der erste Teil der Arbeit erfolgt in Einzelarbeit, der zweite als Gruppengespräch. 
Einzelarbeit: (1) Eine bedeutsame Situation wird beschrieben, wobei auf die Trennung zwischen Beobachtungen und eigenen Gedanken geachtet werden soll (vgl. Keller-Schneider, 2018b, S. 86ff.). (2) Die beschriebene Situation wird anhand der Leitfragen (Abb. 1) analysiert. (3) Die unterschiedlichen Aussagen werden zueinander in Beziehung gesetzt und nach Übereinstimmungen und Widersprüchlichkeiten untersucht. Gruppengespräch: (4) Eine Person stellt ihre Situation und ihre Gedanken dazu vor (Keller-Schneider, 2018b, S. 318f.). Die Gruppenmitglieder hören zu; bei Bedarf können Rückfragen gestellt werden (5). Anschließend bringen die Gruppenmitglieder weitere Deutungsmöglichkeiten und ihre Sichtweisen ein. Diese werden zueinander in Beziehung gesetzt (6), wobei auf Übereinstimmungen und Synergien sowie auf Widersprüchlichkeiten geachtet wird. Aus den herausgearbeiteten Widersprüchlichkeiten werden Handlungsoptionen und Entwicklungsmöglichkeiten abgleitet (7), welche die präsentierende Person abschließend gewichtet (8). Danach wird eine nächste Situation bearbeitet (Schritte 4 bis 8 ).

Bei größeren Gruppen kann nach einer oder nach zwei gemeinsamen Besprechungen die Arbeit in Zweier- oder Dreiergruppen erfolgen. Als Abschluss der Sitzung (9) thematisieren alle Teilnehmenden, was sie zum Weiterdenken angeregt hat und welche Impulse sie mitnehmen.

\section{Ein konkretes Beispiel}

Wie Widersprüchlichkeiten zwischen unterschiedlichen Sichtweisen Entwicklungen anregen, wird anhand der Analyse einer Lehrperson gezeigt (Abb. 2).

Erläuterungen der Lehrerin:

Ich war mit der Stunde sehr zufrieden, merkte zwar, dass ich die Erwartung, dass die Schüler*innen zehn Minuten allein arbeiten sollen, zu hoch gesteckt hatte. Eigentlich wusste ich, dass Zweitklässler nicht ruhig arbeiten können. Doch ich erinnere mich, in der Ausbildung gelernt zu haben, dass es für das Lernen sehr sinnvoll sei, wenn sie zuerst allein arbeiten und sich erst in einem zweiten Schritt austauschen. Dass die Schulleiterin dabei Ruhe erwartete, war mir nicht bewusst, denn ich bin eigentlich der Meinung, dass dies nicht geht. Doch nun muss ich etwas ändern. Kann ich denn von Kindern ein anderes Verhalten erwarten? Doch eigentlich weiß ich nicht, wie ich das machen kann. Ich traue mir das auch nicht zu. 


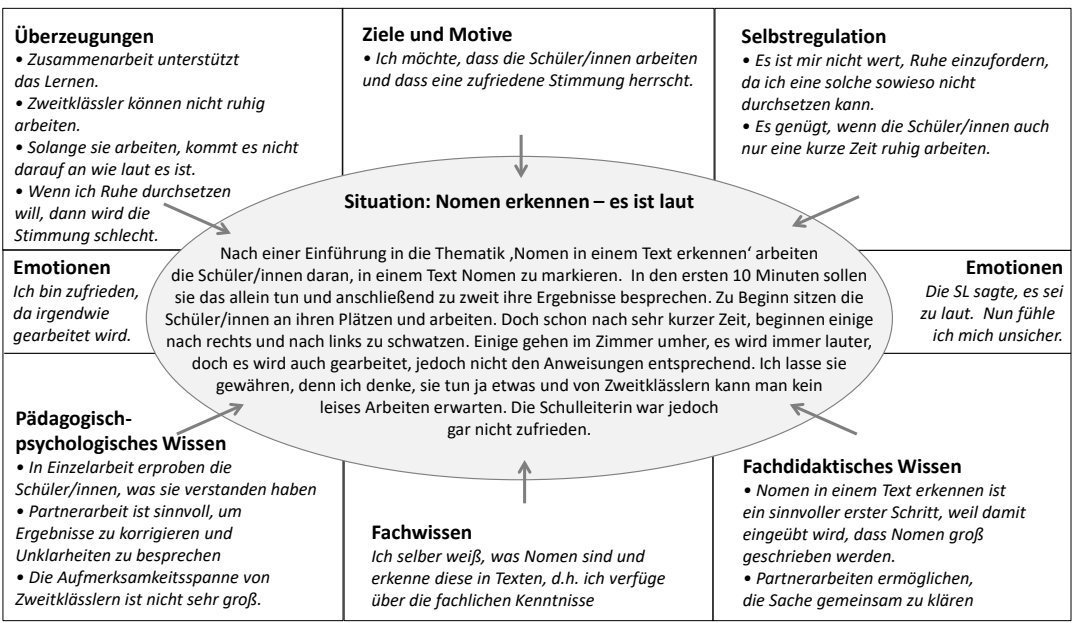

Abb. 2 Analyse einer konkreten Situation (Keller-Schneider, 2018b, S. 47).

Aus den Ausführungen der Lehrerin geht hervor, dass sie ursprünglich mit dem Verlauf dieser Sequenz und dem eingespielten Arbeitsmodus der Klasse zufrieden war. Durch die Rückmeldung der Schulleitung wurde ihre Sicht irritiert, eine Verunsicherung stellte sich ein. Ihre Überzeugung (Zweitklässler können nicht ruhiger arbeiten), welche als Rahmung die Deutung der Arbeitsweise prägte (es ist laut, aber das ist normal bei Zweitklässlern), wurde von der Schulleitung in Frage gestellt. Durch ihre Forderung nach einer Veränderung wurde der Lehrerin klar, dass ihre Sichtweise nicht adäquat ist, dass sie aber nicht weiß, wie sie das ändern kann.

Aus der Analyse geht hervor, dass die Überzeugungen der Schulleiterin und der Lehrperson widersprüchlich sind. Was die Schulleiterin als zu laut deutet, entspricht aus der Sicht der Lehrerin dem, was von Zweitklässlern erwartet werden kann. Ihre Hinweise im Feld der Selbstregulation verweisen darauf, dass sie für das Lenken des Lärmpegels wenig Energie verwendet. Ihre Überzeugung, dass leises Arbeiten nicht möglich sei, unterstützt sie darin. Ihr ist eine gute Stimmung in der Klasse wichtig (Ziele) und dass die Stimmung nicht durch Interventionen der Lehrerin beeinträchtigt werden (Überzeugung). Auch ihr Wissen, dass die Aufmerksamkeitsspanne von Zweitklässlern nicht allzu groß sei, bestätigte sie in ihrem Verhalten in dieser Situation. In dieser Deutung zeigen sich jedoch auch die fließenden Grenzen zwischen Wissen und Überzeugungen, denn die Schullei- 
tung schätzt das Potenzial von Zweitklässlern anders ein. Diese Sichtweise wird von Mitgliedern der Supervisionsgruppe geteilt. Durch die Infragestellung der Überzeugung, dass Zweitklässler nicht ruhig arbeiten können, wird deutlich, dass der Lehrerin Wissen fehlt und dass sie Impulse braucht, um Wege zu finden, wie sie Ruhe schaffen kann. Dass sie sich mit dieser Anforderung befassen muss und auch kann, wird in der Analyse und in der Lösungsfindung in der Gruppe deutlich.

\section{Abschluss}

Dieses Beispiel zeigt auf, wie über Reflexion Grenzen von Überzeugungen und Widersprüchlichkeiten zwischen Zugängen als dynamisierende Komponenten genutzt werden können, um Entwicklungen anzustoßen und sich neuen Ziele zu stellen. In diesem Beispiel wurde der Widerspruch erstmals durch die Rückmeldung der Schulleiterin ausgelöst und von Gruppenmitgliedern geteilt, doch Widersprüchlichkeiten können auch von Gruppenmitgliedern initiiert werden (,Das sehe ich anders, aus meiner Sicht könnte man das auch so deuten ${ }^{\prime}$ ) oder aus eigenen Gedanken hervorgehen (,Das geht doch nicht, doch eigentlich weiß ich nicht, wie ich das ändern kann'). In der Bearbeitung des Reflexionsinstruments wird die Nutzung von mehreren Quellen von Deutungen angeregt, in der Verlangsamung der Gedanken durch das Auffächern auf mehrere Felder wird eine vertiefte Auseinandersetzung angeregt. Zentral ist dabei, dass die analysierte Situation für die individuelle Lehrperson subjektiv von Bedeutung ist und es ihr wichtig ist (internal oder external ausgelöst), sich damit zu beschäftigen. In der mehrmaligen Nutzung dieses mehrperspektivischen Reflexionsinstruments entsteht eine Fähigkeit, Situationen aus unterschiedlichen Perspektiven zu betrachten (reflection on action), auch in der Situation selbst (reflection in action) und ohne den Leitfragen im Einzelnen zu folgen. Aus der Nutzung dieses Reflexionsansatzes resultiert eine adaptive Expertise (Tartwijk et al., 2017), die über Reflexion als dynamisierender Prozess die weitere Professionalisierung unterstützt. 


\section{Literatur}

Berliner, D. C. (2001). Learning about and Learning from Expert Teachers. International Journal of Education Research, 34(5), 463-482.

Blömeke, S., Gustafsson, J. E., Shavelson, R. L. (2015). Beyond dichotomies: Competence viewed as a continuum. Zeitschrift für Psychologie, 223(1), 3-13.

Cherrington, S. (2017). Developing teacher identity through situated cognition approaches to teacher education. In D. J. Clandinin \& J. Husu (Eds.), Handbook of Research on Teacher Education (p. 160-176). London: Sage.

Combe, A., Paseka, A. \& Keller-Schneider, M. (2018). Ungewissheitsdynamiken des Lehrerhandelns. In A. Paseka, M. Keller-Schneider \& A. Combe (Hrsg.), Ungewissheit als Herausforderung für pädagogisches Handeln (S. 53-81). Wiesbaden: VS Springer.

Dreyfus, H. \& Dreyfus, S. (1986). Mind over machine. New York: The Free Press.

Gruber, H. \& Degner, S. (2016). Expertise und Kompetenz. In M. Dick, W. Marotzki \& H. Mieg (Hrsg.), Handbuch Professionsentwicklung (S. 173-180). Bad Heilbrunn: Klinkhardt.

Helsper, W. (2001). Praxis und Reflexion. Journal für Lehrerlnnenbildung, 1(3), 5-17.

Keller-Schneider, M. (2018a). „Es genügt nicht mehr, einfach zu unterrichten“. In A. Paseka, M. Keller-Schneider \& A. Combe (Hrsg.), Ungewissheit als Herausforderung für pädagogisches Handeln (S. 231-254). Wiesbaden: Springer VS.

Keller-Schneider, M. (2018b). Impulse zum Berufseinstieg von Lehrpersonen. Grundlagen - Erfahrungsberichte - Reflexionsinstrumente. Bern: hep.

Keller-Schneider, M. (2020a). Entwicklungsaufgaben im Berufseinstieg von Lehrpersonen (2., überarbeitete und erweiterte Aufl.) Münster: Waxmann.

Keller-Schneider, M. (2020b). Was beschäftigt Studierende in der Rolle als Lehrpersonen? Jahrbuch für Allgemeine Didaktik, 10(1), 40-58.

Korthagen, F. (2014). Promoting core reflection in teacher education. In C. J. Craig \& L. Orland (Eds.), International Teacher Education (p. 73-79). London: Emerald Group.

Lazarus, R. S. \& Folkman, S. (1984). Stress, Appraisal and Coping. New York: Springer.

Mandl, H. \& Gerstenmaier, J. (2000). Die Kluft zwischen Wissen und Handeln. Göttingen: Hogrefe.

Neuweg, H. G. (2014). Das Wissen der Wissensvermittler. In E. Terhart, H. Bennewitz \& M. Rothland (Hrsg.), Handbuch der Forschung zum Lehrerberuf (S. 583-614). Münster: Waxmann.

Nguyen, Q., Fernandez, N., Karsenti, T. \& Charlin, B. (2014). What is reflection? Medical Education, 48, 1176-1189.

Schön, D. A. (1983). The reflective practitioner. New York: Basic Books.

Tartwijk, J., Zwart, R. \& Wubbels, T. (2017). Developing teachers' competences with the focus on adaptive expertise in teaching. In D. J. Clandinin \& J. Husu (Eds.), Handbook of Research on Teacher Education (p. 820-833). London: Sage. 
Manuela Keller-Schneider, Prof. Dr. phil., Pädagogische Hochschule Zürich. Arbeitsschwerpunkte: Professionsforschung, insbesondere Berufseinstieg, Team und Schulentwicklung, selbstreguliertes Lernen

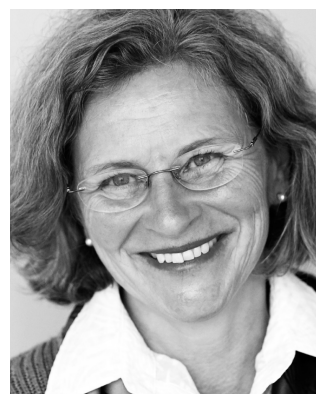

